
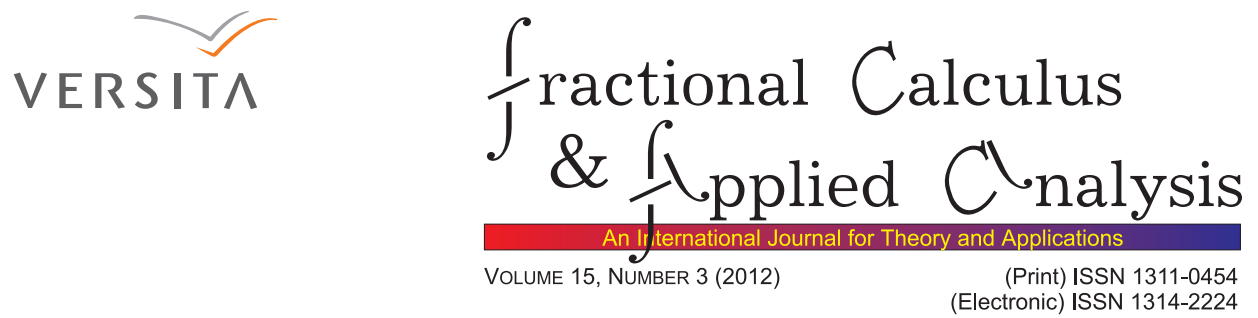

RESEARCH PAPER

\title{
PRODUCT RULE FOR VECTOR FRACTIONAL DERIVATIVES
}

\section{Diogo Bolster $^{1}$, Mark M. Meerschaert ${ }^{2}$, Alla Sikorskii ${ }^{3}$}

\begin{abstract}
This paper establishes a product rule for fractional derivatives of a realvalued function defined on a finite dimensional Euclidean vector space. The proof uses Fourier transforms.

MSC 2010: Primary: 26A33, 26B12; Secondary: 60E07

Key Words and Phrases: fractional derivative, vector fractional derivative, Fourier transform, fractional advection-dispersion equation

Editorial Note: Information about the recent authors' book 13] (by M.M. Meerschaert, A. Sikorskii), is given in this issue's Editorial Note.
\end{abstract}

\section{Introduction}

The purpose of this paper is to prove a product rule for vector fractional derivatives. The product rule for scalar fractional derivatives is known. The proof in Podlubny [15] uses a finite difference approach, and the proof in Osler [14] uses the Cauchy integral formula. We provide a simple proof using Fourier transforms, and then extend to vector fractional derivatives, using the same approach.

The one dimensional Fourier transform

$$
\hat{f}(k)=\int_{-\infty}^{\infty} e^{-i k x} f(x) d x
$$

(c) 2012 Diogenes Co., Sofia

pp. $463-478$, DOI: $10.2478 / \mathrm{s} 13540-012-0033-0$ 
is defined for any integrable function $f(x)$, i.e., a function such that

$$
\int_{-\infty}^{\infty}|f(x)| d x<\infty
$$

If $f(x), \mathbb{D} f(x)=f^{\prime}(x), \ldots, \mathbb{D}^{n} f(x)$ are all integrable, then it is well known (e.g., see [13, Section 1.1]) that $(i k)^{n} \hat{f}(k)$ is the Fourier transform of the $n$th derivative $\mathbb{D}^{n} f(x)$. Then it is natural to define the fractional derivative $\mathbb{D}^{\alpha} f(x)$ as the function with Fourier transform $(i k)^{\alpha} \hat{f}(k)$ for some $\alpha>0$. The fractional derivative can also be defined as the limit of a difference quotient. The first derivative is

$$
\mathbb{D} f(x)=f^{\prime}(x)=\lim _{h \rightarrow 0} \frac{f(x)-f(x-h)}{h}
$$

when the limit exists. Higher order derivatives are defined by

$$
\mathbb{D}^{n} f(x)=\lim _{h \rightarrow 0} \frac{\Delta^{n} f(x)}{h^{n}}
$$

where

$$
\begin{aligned}
\Delta f(x) & =f(x)-f(x-h) \\
\Delta^{2} f(x) & =\Delta \Delta f(x)=\Delta[f(x)-f(x-h)] \\
& =f(x)-2 f(x-h)+f(x-2 h) \\
\Delta^{3} f(x) & =f(x)-3 f(x-h)+3 f(x-2 h)-f(x-3 h) \\
\vdots & \\
\Delta^{n} f(x) & =\sum_{j=0}^{n}\left(\begin{array}{l}
n \\
j
\end{array}\right)(-1)^{j} f(x-j h)
\end{aligned}
$$

using the binomial formula. The fractional difference operator

$$
\Delta^{\alpha} f(x)=\sum_{j=0}^{\infty}\left(\begin{array}{l}
\alpha \\
j
\end{array}\right)(-1)^{j} f(x-j h)
$$

where

$$
\left(\begin{array}{c}
\alpha \\
j
\end{array}\right)=\frac{\Gamma(\alpha+1)}{j ! \Gamma(\alpha-j+1)}
$$

extends the usual definition, since $\Gamma(n+1)=n$ ! for positive integers $n$. Then the Grünwald-Letnikov fractional derivative can be defined as

$$
\mathbb{D}^{\alpha} f(x)=\lim _{h \rightarrow 0} \frac{\Delta^{\alpha} f(x)}{h^{\alpha}}
$$

whenever the limit exists. If $f(x)$ is bounded, and if $f(x)$ and its derivatives up to order $n>1+\alpha$ are integrable, then [13, Proposition 2.1] implies that 
the Grünwald fractional derivative (1.1) exists, and its Fourier transform also exists, and equals $(i k)^{\alpha} \hat{f}(k)$.

Some alternative forms of the fractional derivative are also useful in applications. If $0<\alpha<1$, then an explicit computation [13, Proposition 3.10 ] shows that

$$
(i k)^{\alpha}=\frac{1}{\Gamma(1-\alpha)} \int_{0}^{\infty}\left(1-e^{-i k y}\right) \alpha y^{-\alpha-1} d y .
$$

Invert the Fourier transform $(i k)^{\alpha} \hat{f}(k)$, and use the fact that

$$
\int e^{-i k x} f(x-y) d x=e^{-i k y} \hat{f}(k)
$$

to see that

$$
\mathbb{D}^{\alpha} f(x)=\int_{0}^{\infty}[f(x)-f(x-y)] \frac{\alpha}{\Gamma(1-\alpha)} y^{-\alpha-1} d y .
$$

This is called the generator form of the fractional derivative. If $f, f^{\prime}$ are integrable, and vanish at infinity, then the generator form of the fractional derivative exists and has Fourier transform $(i k)^{\alpha} \hat{f}(k)$, see [13, Example 3.24]. For $1<\alpha<2$, a computation [13, Proposition 3.12] shows that

$$
(i k)^{\alpha}=\frac{\alpha-1}{\Gamma(2-\alpha)} \int_{0}^{\infty}\left(e^{-i k y}-1+i k y\right) \alpha y^{-\alpha-1} d y .
$$

Then it follows that

$$
\mathbb{D}^{\alpha} f(x)=\frac{\alpha(\alpha-1)}{\Gamma(2-\alpha)} \int_{0}^{\infty}\left[f(x-y)-f(x)+y f^{\prime}(x)\right] y^{-1-\alpha} d y
$$

If $1<\alpha<2$, and if $f, f^{\prime}, f^{\prime \prime}$ are integrable and vanish at infinity, then the generator form of the fractional derivative exists and has Fourier transform $(i k)^{\alpha} \hat{f}(k)$, see [13, Theorem 3.17, Example 3.25].

One important application of fractional derivatives is the fractional advection-dispersion equation (FADE)

$$
\frac{\partial C}{\partial t}=-v \frac{\partial C}{\partial x}+a \frac{\partial^{\alpha} C}{\partial x^{\alpha}}
$$

that models the flow and transport of pollutants in underground water. Here $C=C(x, t)$ represents the concentration of pollutants at time $t>0$ at location $x \in \mathbb{R}$, and $\partial^{\alpha} / \partial x^{\alpha}=\mathbb{D}_{x}^{\alpha}$, the fractional derivative $\mathbb{D}^{\alpha}$ applied to the $x$ variable. See Benson et al. [2] for more details. 


\section{The one variable case}

The product rule states that if $f(x)$ and $g(x)$ are two differentiable functions, then

$$
\mathbb{D}[f(x) g(x)]=[f g]^{\prime}=f^{\prime} g+f g^{\prime}=\mathbb{D}[f(x)] g(x)+f(x) \mathbb{D}[g(x)] .
$$

For higher order derivatives, the product rule follows by repeated application of the first derivative formula:

$$
\begin{aligned}
\mathbb{D}^{2}[f(x) g(x)] & =\left[\mathbb{D}^{2} f(x)\right] g(x)+2[\mathbb{D} f(x)][\mathbb{D} g(x)]+f(x)\left[\mathbb{D}^{2} g(x)\right] \\
\mathbb{D}^{3}[f(x) g(x)] & =\left[\mathbb{D}^{3} f(x)\right] g(x)+3\left[\mathbb{D}^{2} f(x)\right][\mathbb{D} g(x)]+3[\mathbb{D} f(x)]\left[\mathbb{D}^{2} g(x)\right] \\
& +f(x)\left[\mathbb{D}^{3} g(x)\right] \\
& \vdots \\
\mathbb{D}^{n}[f(x) g(x)] & =\sum_{j=0}^{n}\left(\begin{array}{c}
n \\
j
\end{array}\right)\left[\mathbb{D}^{n-j} f(x)\right]\left[\mathbb{D}^{j} g(x)\right] .
\end{aligned}
$$

A fractional product rule can be found in the book of Podlubny [15]:

$$
\mathbb{D}^{\alpha}[f(x) g(x)]=\sum_{j=0}^{\infty}\left(\begin{array}{l}
\alpha \\
j
\end{array}\right)\left[\mathbb{D}^{\alpha-j} f(x)\right]\left[\mathbb{D}^{j} g(x)\right] .
$$

When $j>\alpha$, the term $\mathbb{D}^{\alpha-j} f(x)$ is called a fractional integral and is also denoted by $\mathbb{I}^{j-\alpha} f(x)$. The fractional integral is defined as

$$
\mathbb{I}^{j-\alpha} f(x)=\frac{1}{\Gamma(j-\alpha)} \int_{-\infty}^{x} f(u)(x-u)^{j-\alpha-1} d u .
$$

The proof in Podlubny [15] uses an expansion of the Grünwald formula (1.1). A different proof, using the Cauchy integral formula, is given in Osler [14]. Liouville [9] proved (2.1) using Fourier series, while Grünwald [4] and Letnikov [8] proved (2.1) directly from the definition of $\mathbb{I}^{j-\alpha} f(x)$. This paper provides an alternative proof, using Fourier transforms. Then in Section 3, the product rule (2.1) is extended to vector fractional derivatives, using the same approach.

First note that the Fourier transform of a product is (up to a constant) a convolution:

$$
\widehat{f g}(k)=\int_{-\infty}^{\infty} e^{-i k x} f(x) g(x) d x=\frac{1}{2 \pi} \hat{f}(k) * \hat{g}(k) .
$$

To see this, apply the Fourier inversion formula [13, Theorem 1.4]: If $\int|h(x)| d x<\infty$, the Fourier transform $\hat{h}(k)$ exists. If $\int|\hat{h}(k)| d k<\infty$ 
as well, then

$$
h(x)=\frac{1}{2 \pi} \int e^{i k x} \hat{h}(k) d k
$$

for all $x \in \mathbb{R}$. Now we will apply this result to the function $h(x)=f(x) g(x)$. A substitution $z=k-y$ shows that the inverse Fourier transform of

$$
\frac{1}{2 \pi}[\hat{f}(k) * \hat{g}(k)]=\frac{1}{2 \pi} \int_{-\infty}^{\infty} \hat{f}(k-y) \hat{g}(y) d y
$$

is

$$
\begin{aligned}
& \left(\frac{1}{2 \pi}\right)^{2} \int_{-\infty}^{\infty} e^{i k x}\left(\int_{-\infty}^{\infty} \hat{f}(k-y) \hat{g}(y) d y\right) d k \\
& =\left(\frac{1}{2 \pi}\right)^{2} \int_{-\infty}^{\infty} e^{i(z+y) x} \int_{-\infty}^{\infty} \hat{f}(z) \hat{g}(y) d y d z \\
& =\frac{1}{2 \pi} \int_{-\infty}^{\infty} e^{i z x} \hat{f}(z) d z \frac{1}{2 \pi} \int_{-\infty}^{\infty} e^{i y x} \hat{g}(y) d y \\
& =f(x) g(x) .
\end{aligned}
$$

Then under certain conditions (see Theorem 2.1), the fractional derivative $\mathbb{D}^{\alpha}[f(x) g(x)]$ can be defined as the function with Fourier transform $(i k)^{\alpha} \frac{1}{2 \pi}[\hat{f}(k) * \hat{g}(k)]$.

We will prove the fractional product rule in the sense of tempered distributions, which is the natural setting for Fourier transforms. Recall that the space of rapidly decreasing functions $S\left(\mathbb{R}^{d}\right)$ consists of the infinitely differentiable functions $g: \mathbb{R}^{d} \rightarrow \mathbb{R}$ such that

$$
\sup _{x \in \mathbb{R}^{d}}\left|x^{n} \mathbb{D}_{x}^{m} g(x)\right|<\infty
$$

where $n=\left(n_{1}, \ldots, n_{d}\right)$ and $m=\left(m_{1}, \ldots, m_{d}\right)$ are vectors of non-negative integers, and

$$
x^{n}=x_{1}^{n_{1}} \cdots x_{d}^{n_{d}}, \text { and } \mathbb{D}_{x}^{m}=\mathbb{D}_{x_{1}}^{m_{1}} \cdots \mathbb{D}_{x_{d}}^{m_{d}} .
$$

The space $S^{\prime}\left(\mathbb{R}^{d}\right)$ of continuous linear functionals on $S\left(\mathbb{R}^{d}\right)$ is called the space of tempered distributions. The Fourier transform, and inverse Fourier transform, are linear continuous mappings of $S^{\prime}\left(\mathbb{R}^{d}\right)$ into itself. If $f: \mathbb{R}^{d} \rightarrow$ $\mathbb{R}$ is of polynomial growth, meaning that

$$
\int|f(x)|(1+|x|)^{-p} d x<\infty
$$

for some $p>0$, then $T_{f}(\varphi)=\int f(x) \varphi(x) d x:=\langle f, \varphi\rangle$ is a tempered distribution, also called a generalized function. The Fourier transform of this 
generalized function is defined as $\hat{T}_{f}(\varphi)=\langle\hat{f}, \varphi\rangle=\langle f, \hat{\varphi}\rangle=T_{f}(\hat{\varphi})$ for $\varphi \in S\left(\mathbb{R}^{d}\right)$. See Yosida [19, Ch.VI] for more details. If $f$ is a tempered distribution, then the fractional integral $\mathbb{I}^{j-\alpha} f(x)$ always exists as the convolution with the tempered distribution $x^{j-\alpha-1} I(x>0) / \Gamma(j-\alpha)$.

Theorem 2.1. Suppose that $f(x) g(x)$ is integrable and has a continuous integrable first derivative that vanishes at infinity if $0<\alpha<1$, or continuous integrable first and second derivatives that vanish at infinity if $1<\alpha<2$. Suppose that $\mathbb{D}^{\alpha-j} f(x)$ exists and is of polynomial growth for every integer $j \geq 0, g \in S(\mathbb{R})$, and the series

$$
\sum_{j=0}^{\infty}\left(\begin{array}{c}
\alpha \\
j
\end{array}\right) \mathbb{D}^{\alpha-j} f \mathbb{D}^{j} g
$$

converges in $S^{\prime}(\mathbb{R})$. Then (2.1) holds in the sense of generalized functions. If the series (2.4) converges pointwise, then (2.1) holds pointwise.

P r o o f. The binomial series

$$
(1+z)^{\alpha}=\sum_{j=0}^{\infty}\left(\begin{array}{l}
\alpha \\
j
\end{array}\right) z^{j}
$$

converges for any complex $|z| \leq 1$ and any $\alpha>0$ (e.g., see Hille [6, p. $147]$ ). Since this is a power series, the convergence is uniform on $|z| \leq 1$. It follows that

$$
(z+w)^{\alpha}=\sum_{j=0}^{\infty}\left(\begin{array}{l}
\alpha \\
j
\end{array}\right) z^{\alpha-j} w^{j}
$$

for any complex numbers $z, w$. To see this, suppose (without any loss of generality) that $|w| \leq|z|$. Then we may write

$$
\begin{aligned}
(z+w)^{\alpha} & =z^{\alpha}(1+(w / z))^{\alpha} \\
& =z^{\alpha} \sum_{j=0}^{\infty}\left(\begin{array}{l}
\alpha \\
j
\end{array}\right)(w / z)^{j} \\
& =\sum_{j=0}^{\infty}\left(\begin{array}{c}
\alpha \\
j
\end{array}\right) z^{\alpha-j} w^{j}
\end{aligned}
$$

which agrees with (2.6) .

Under the theorem assumptions, $f(x) g(x)$ and its derivatives (of order one if $0<\alpha<1$, or up to order two if $1<\alpha<2$ ) are continuous and integrable and vanish at infinity. Then the fractional derivative $\mathbb{D}^{\alpha}[f(x) g(x)]$ can be defined as the function with Fourier transform $(i k)^{\alpha}(2 \pi)^{-1}[\hat{f}(k) * \hat{g}(k)]$, see for example [13, Examples 3.24-3.25]. Then 
the generalized function $\mathbb{D}^{\alpha}[f(x) g(x)]$ defines a linear functional on $S(\mathbb{R})$ such that

$$
\left\langle\mathbb{D}^{\alpha}[f g], \varphi\right\rangle=\int_{-\infty}^{\infty} \mathbb{D}^{\alpha}[f(x) g(x)] \varphi(x) d x
$$

for any $\varphi \in S(\mathbb{R})$. Its Fourier transform satisfies

$$
\left\langle\widehat{\mathbb{D}^{\alpha}[f g]}, \varphi\right\rangle=\int_{-\infty}^{\infty} \widehat{\mathbb{D}^{\alpha}[f g]}(x) \varphi(x) d x=\int_{-\infty}^{\infty} \mathbb{D}^{\alpha}[f g](x) \hat{\varphi}(x) d x
$$

and hence

$$
\begin{aligned}
\left\langle\widehat{\mathbb{D}^{\alpha}[f g]}, \varphi\right\rangle & =\int_{-\infty}^{\infty}(i k)^{\alpha}\left[\frac{\hat{f}(k) * \hat{g}(k)}{2 \pi}\right] \varphi(k) d k \\
& =\int_{-\infty}^{\infty} \frac{1}{2 \pi} \int_{-\infty}^{\infty}(i k)^{\alpha} \hat{f}(k-y) \hat{g}(y) \varphi(k) d y d k \\
& =\int_{-\infty}^{\infty} \frac{1}{2 \pi} \int_{-\infty}^{\infty}\{(i k-i y)+(i y)\}^{\alpha} \hat{f}(k-y) \hat{g}(y) \varphi(k) d y d k \\
& =\frac{1}{2 \pi} \int_{-\infty}^{\infty} \int_{-\infty}^{\infty} \sum_{j=0}^{\infty}\left(\begin{array}{c}
\alpha \\
j
\end{array}\right)(i k-i y)^{\alpha-j}(i y)^{j} \hat{f}(k-y) \hat{g}(y) \varphi(k) d y d k
\end{aligned}
$$

The series

$$
\sum_{j=0}^{\infty}\left(\begin{array}{c}
\alpha \\
j
\end{array}\right)(i k-i y)^{\alpha-j}(i y)^{j}
$$

converges uniformly, and can be integrated term by term with respect to $F(d y, d k)=\hat{f}(k-y) \hat{g}(y) \varphi(k) d y d k$, see [17, Theorem 7.16]. Then it follows that

$$
\begin{aligned}
\left\langle\widehat{\mathbb{D}^{\alpha}[f g]}, \varphi\right\rangle & =\sum_{j=0}^{\infty}\left(\begin{array}{c}
\alpha \\
j
\end{array}\right) \frac{1}{2 \pi} \int_{-\infty}^{\infty} \int_{-\infty}^{\infty}(i k-i y)^{\alpha-j} \hat{f}(k-y)(i y)^{j} \hat{g}(y) \varphi(k) d y d k \\
& =\sum_{j=0}^{\infty}\left(\begin{array}{c}
\alpha \\
j
\end{array}\right) \frac{1}{2 \pi} \int_{-\infty}^{\infty} \int_{-\infty}^{\infty}(i z)^{\alpha-j} \hat{f}(z)(i(k-z))^{j} \hat{g}(k-z) \varphi(k) d z d k \\
& =\sum_{j=0}^{\infty}\left(\begin{array}{c}
\alpha \\
j
\end{array}\right) \frac{1}{2 \pi} \int_{-\infty}^{\infty} \int_{-\infty}^{\infty} \widehat{\mathbb{D}^{\alpha-j}} f(z)(i(k-z))^{j} \hat{g}(k-z) \varphi(k) d k d z
\end{aligned}
$$

The $j$ th integral term is the action of $\widehat{\mathbb{D}^{\alpha-j} f}$ on the function

$$
\int_{-\infty}^{\infty}(i(k-z))^{j} \hat{g}(k-z) \varphi(k) d k,
$$

This function belongs to $S(\mathbb{R})$, since $S(\mathbb{R})$ is closed under convolutions, and since $z^{j} \hat{g}(z) \in S(\mathbb{R})$, in view of the fact that $g \in S(\mathbb{R})$. The Fourier transform of the generalized function $\mathbb{D}^{\alpha-j} f(x)$ is $(i k)^{\alpha-j} \hat{f}(k)$, and $(i k)^{j} \hat{g}(k)$ is 
the pointwise Fourier transform of $\mathbb{D}^{j} g(x)$, since $g \in S(\mathbb{R})$. Then it follows from (2.2) that the convolution

$$
\frac{1}{2 \pi} \int_{-\infty}^{\infty}(i k-i y)^{\alpha-j} \hat{f}(k-y)(i y)^{j} \hat{g}(y) d y
$$

is the Fourier transform of the generalized function $\mathbb{D}^{\alpha-j} f(x) \mathbb{D}^{j} g(x)$. Hence

$$
\left\langle\widehat{\mathbb{D}^{\alpha}[f g]}, \varphi\right\rangle=\sum_{j=0}^{\infty}\left(\begin{array}{c}
\alpha \\
j
\end{array}\right)\left\langle{\mathbb{\mathbb { D } ^ { \alpha - j f \mathbb { D } }} j}^{j}, \varphi\right\rangle,
$$

and

$$
\left\langle\mathbb{D}^{\alpha}[f g], \hat{\varphi}\right\rangle=\sum_{j=0}^{\infty}\left(\begin{array}{c}
\alpha \\
j
\end{array}\right)\left\langle\mathbb{D}^{\alpha-j} f \mathbb{D}^{j} g, \hat{\varphi}\right\rangle
$$

which agrees with (2.1), provided that (2.4) holds.

Now suppose that the series (2.4) converges pointwise. For any fixed $y$, define $\hat{\varphi}(x)=\omega_{\epsilon}(x-y)$, where the approximate identity

$$
\omega_{\epsilon}(x)=c_{\epsilon} \exp \left\{-\epsilon^{2} /\left(\epsilon^{2}-x^{2}\right)\right\} 1_{\{|x|<\epsilon\}},
$$

and the constant $c_{\epsilon}$ is chosen so that $\int \omega_{\epsilon}(x) d x=1$. Then for any locally integrable function $h(x), \int \omega_{\epsilon}(x-y) h(y) d y$ converges to $h(x)$ uniformly on compact sets as $\epsilon \rightarrow 0$. Since (2.7) holds for every $\hat{\varphi}(x)=\omega_{\epsilon}(x-y)$, it follows that the product rule (2.1) holds pointwise.

REMARK 2.1. An alternative proof of Theorem 2.1 can be written using pointwise Fourier transforms, and the Fourier inversion formula. However, to apply this result, it is required that $\mathbb{D}^{\alpha-j} f(x)$ and $\mathbb{D}^{j} g(x)$ are integrable for every $j \geq 0$. There are few (if any) practical examples of functions $f(x)$ whose (fractional) integrals exist and are integrable for all $j \geq 0$. Consider for example the step function $f(x)=1_{\{0 \leq|x| \leq 1\}}$ (or a smooth approximation of this function). For this reason, we use generalized functions, and then we only require that $\mathbb{D}^{\alpha-j} f(x)$ is of polynomial growth for every non-negative integer $j$. The interpretation of fractional derivatives and Fourier transforms as generalized functions is common in applications, see for example [5, 7, 18].

REMARK 2.2. A different proof of Theorem 2.1 can be written using Laplace transforms. This only requires that $\mathbb{D}^{\alpha-j} f$ and $\mathbb{D}^{j} g$ are exponentially bounded, so that the Laplace transforms exist. The proof is quite similar to Theorem 2.1, after smoothing so that $f, g$ and all their derivatives vanish at at $x=0$. However, for applications to functions on $\mathbb{R}^{1}$ or $\mathbb{R}^{d}$, the Fourier approach is most useful. 
EXAmple 2.1. For an explicit example where Theorem 2.1 applies, take $f(x)=x^{p} I(x>0)$ for some $p>\alpha$, and $g(x)=e^{-b x} I(x>0)$ for some $b>0$. An explicit computation shows that

$$
\mathbb{D}^{\alpha-j} f(x)=\frac{\Gamma(p+1)}{\Gamma(p+1+j-\alpha)} x^{p+\alpha-j} I(x>0)
$$

and of course $\mathbb{D}^{j} g(x)=(-b)^{j} e^{-b x} I(x>0)$. Then all the conditions of Theorem 2.1 are satisfied (using a smoothed version of $g(x)$ at $x=0$ ), and the series (2.4) converges pointwise, so that the product rule (2.1) holds pointwise.

ExAmPle 2.2. The product rule for fractional derivatives has been applied to chemical mixing in a heterogeneous porous medium [3]. In a simple chemical reaction $C_{1}+C_{2} \rightarrow C_{3}$, the local concentrations $c_{i}(x, t)$ satisfy a coupled system of space-fractional reaction-diffusion equations:

$$
\begin{aligned}
& \frac{\partial c_{i}(x, t)}{\partial t}=-v \frac{\partial c_{i}(x, t)}{\partial x}+a \frac{\partial^{\alpha} c_{i}(x, t)}{\partial x^{\alpha}}+r(x, t) \quad \text { for } i=1,2, \\
& \frac{\partial c_{3}(x, t)}{\partial t}=-r(x, t)
\end{aligned}
$$

since the precipitant $C_{3}$ remains immobile. The reactants are in local equilibrium: $c_{1} c_{2}=K$. Then the local concentration difference $u=c_{1}-c_{2}$ satisfies the conservative FADE (1.4). Note from (2.8) that the local reaction rate is

$$
r=\frac{\partial c_{1}}{\partial t}+v \frac{\partial c_{1}}{\partial x}-a \frac{\partial^{\alpha} c_{1}}{\partial x^{\alpha}}
$$

Write $u=c_{1}-K / c_{1}$ and solve for $c_{1}$ as a function of $u$. Use the chain rule to write

$$
r=\frac{\partial c_{1}}{\partial u} \frac{\partial u}{\partial t}+v \frac{\partial c_{1}}{\partial u} \frac{\partial u}{\partial x}-a \mathbb{D}_{x}^{\alpha-1}\left[\frac{\partial c_{1}}{\partial u} \frac{\partial u}{\partial x}\right]
$$

using the fact that $\mathbb{D}^{\alpha}=\mathbb{D}^{\alpha-1} \mathbb{D}^{1}$. Using the semi-analytic solution to the constant coefficient FADE (1.4) for $u$ in terms of a smooth $\alpha$-stable probability density function [2], an explicit solution for the local reaction rate can be obtained using the fractional product rule (2.1). To apply Theorem 2.1 with $f=\partial c_{1} / \partial u$ and $g=\partial u / \partial x$, approximate the stable density $u(x)$ by a tempered stable $[1,16$, so that $g \in S(\mathbb{R})$, and then let the tempering constant go to zero.

\section{Vector fractional derivatives}

Suppose that $f(x)=f\left(x_{1}, \ldots, x_{d}\right)$ is a scalar-valued function of the vector argument $x=\left(x_{1}, \ldots, x_{d}\right)^{\prime}$ in $d$ dimensions. Its Fourier transform is 
defined by

$$
\hat{f}(k)=\int e^{-i k \cdot x} f(x) d x,
$$

where $k=\left(k_{1}, \ldots, k_{d}\right)^{\prime}$ is also a $d$-dimensional vector, and

$$
k \cdot x=k^{\prime} x=\left(k_{1}, \ldots, k_{d}\right)\left(\begin{array}{c}
x_{1} \\
\vdots \\
x_{d}
\end{array}\right)=\sum_{j=1}^{d} k_{j} x_{j}
$$

is the scalar product (dot product, inner product). The gradient

$$
\nabla f(x)=\left(\begin{array}{c}
\frac{\partial}{\partial x_{1}} f\left(x_{1}, \ldots, x_{d}\right) \\
\vdots \\
\frac{\partial}{\partial x_{d}} f\left(x_{1}, \ldots, x_{d}\right)
\end{array}\right)
$$

is a vector-valued function whose Fourier transform is

$$
(i k) \hat{f}(k)=\int e^{-i k \cdot x} \nabla f(x) d x
$$

where the integral is defined componentwise. The proof is a direct application of the corresponding one dimensional formula.

The product rule for two such functions $f, g$ is

$$
\begin{aligned}
\nabla[f(x) g(x)] & =\left(\begin{array}{c}
\frac{\partial}{\partial x_{1}}[f(x) g(x)] \\
\vdots \\
\frac{\partial}{\partial x_{d}}[f(x) g(x)]
\end{array}\right) \\
& =\left(\begin{array}{c}
{\left[\frac{\partial}{\partial x_{1}} f(x)\right] g(x)+f(x)\left[\frac{\partial}{\partial x_{1}} g(x)\right]} \\
\vdots \\
{\left[\frac{\partial}{\partial x_{d}} f(x)\right] g(x)+f(x)\left[\frac{\partial}{\partial x_{d}} g(x)\right]}
\end{array}\right) \\
& =[\nabla f(x)] g(x)+f(x)[\nabla g(x)] .
\end{aligned}
$$

The Laplacian

$$
\Delta f(x)=\nabla \cdot \nabla f(x)=\sum_{j=1}^{d} \frac{\partial^{2}}{\partial x_{j}^{2}} f(x)
$$

is a scalar-valued function whose Fourier transform is $(i k) \cdot(i k) \hat{f}(k)=$ $-\|k\|^{2} \hat{f}(k)$. It is also customary to write $\Delta f(x)=\nabla^{2} f(x)$ by an abuse of 
notation. The product rule for the Laplacian is

$$
\begin{aligned}
\Delta[f(x) g(x)] & =\nabla \cdot \nabla[f(x) g(x)] \\
& =\nabla \cdot\{[\nabla f(x)] g(x)+f(x) \nabla g(x)\} \\
& =\left[\nabla^{2} f(x)\right] g(x)+2[\nabla f(x)] \cdot[\nabla g(x)]+f(x)[\nabla g(x)] \\
& =\sum_{j=0}^{2}\left(\begin{array}{l}
2 \\
j
\end{array}\right) \nabla^{2-j} f(x) \cdot \nabla^{j} g(x) .
\end{aligned}
$$

The scalar-valued fractional derivative $\mathbb{D}_{M}^{\alpha} f(x)$ can be defined as the function with Fourier transform

$$
\int_{\|\theta\|=1}(i k \cdot \theta)^{\alpha} \hat{f}(k) M(d \theta)
$$

where $M(d \theta)$ is an arbitrary (positive) Borel measure on the unit sphere, and $\alpha \in \mathbb{R}$. When $M$ is uniform, $\mathbb{D}_{M}^{\alpha} f(x)$ reduces to a constant multiple of the fractional Laplacian. The fractional Laplacian $\Delta^{\alpha / 2} f(x)$ can be defined as the function with Fourier transform $-\|k\|^{\alpha} \hat{f}(k)$. When $\alpha=2$, this reduces to the traditional Laplacian. The vector-valued fractional gradient $\nabla_{M}^{\alpha} f(x)$ can be defined as the function with Fourier transform

$$
\int_{\|\theta\|=1} \theta(i k \cdot \theta)^{\alpha} \hat{f}(k) M(d \theta)
$$

If $\alpha=1$ and the mixing measure $M$ assigns unit mass to the positive coordinate vectors, then this reduces to the traditional gradient. Using these Fourier transform definitions, it is easy to check that $\mathbb{D}_{M}^{\alpha} f(x)=$ $\nabla_{M}^{\alpha-1} \cdot \nabla f(x)$. See [12] for more details.

The fractional derivative $\mathbb{D}_{M}^{\alpha} f(x)$ is a weighted average of fractional directional derivatives. The directional derivative

$$
\mathbb{D}_{\theta}^{1} f(x)=\theta \cdot \nabla f(x)=\left.\frac{d}{d t} f(x+\theta t)\right|_{t=0}
$$

has Fourier transform $(i k \cdot \theta) \hat{f}(k)$. The fractional directional derivative can be defined by

$$
\mathbb{D}_{\theta}^{\alpha} f(x)=\left.\mathbb{D}^{\alpha} f(x+\theta t)\right|_{t=0},
$$

where the fractional derivative $\mathbb{D}^{\alpha}$ is taken with respect to the variable $t \in$ $\mathbb{R}$. Using the generator form (1.2) of the fractional derivative for $0<\alpha<1$, or the form (1.3) for $1<\alpha<2$, it is not hard to check [13, Section 6.5] that $\mathbb{D}_{\theta}^{\alpha} f(x)$ has Fourier transform $(i k \cdot \theta)^{\alpha} \hat{f}(k)$. Then we can also write

$$
\mathbb{D}_{M}^{\alpha} f(x)=\int_{\|\theta\|=1} \mathbb{D}_{\theta}^{\alpha} f(x) M(d \theta) .
$$


For $0<\alpha<1$ or $1<\alpha<2$, this formula is valid when $f$ and all of its partial derivatives up to order two exist, are integrable, and vanish at infinity, see Examples 6.28-6.29 in [13]. A very similar argument shows that

$$
\nabla_{M}^{\alpha} f(x)=\int_{\|\theta\|=1} \theta \mathbb{D}_{\theta}^{\alpha} f(x) M(d \theta) .
$$

When $j>\alpha$, the fractional integral

$$
\mathbb{I}_{\theta}^{j-\alpha} f(x)=\mathbb{I}^{j-\alpha} f(x+t \theta),
$$

where we integrate with respect to the variable $t \in \mathbb{R}$, and

$$
\mathbb{D}_{M}^{\alpha-j} f(x)=\int_{\|\theta\|=1} \mathbb{I}_{\theta}^{j-\alpha} f(x) M(d \theta)
$$

The multi-variable FADE model for pollution migration is

$$
\frac{\partial C}{\partial t}=-v \cdot \nabla C+a \mathbb{D}_{M}^{\alpha} C
$$

where $1<\alpha<2, C=C(x, t)$ is the pollution concentration at location $x \in \mathbb{R}^{d}$ at time $t>0, v \in \mathbb{R}^{d}$ is the velocity vector that gives the average velocity of the pollution plume, and $a>0$ is a constant. Note that we can also write

$$
\frac{\partial C}{\partial t}=-v \cdot \nabla C+a \nabla_{M}^{\alpha-1} \cdot \nabla C
$$

in which the dispersive term is the fractional divergence of the concentration gradient [12. The fractional partial differential equation (3.3) governs the long-time limit of a random walk with jumps $X=R \Theta$, where we assume $\mathbb{P}(R>r) \sim C r^{-\alpha}$ and $\mathbb{P}(\Theta \in B)=M(B)$ for any finite Borel subset $B$ of the unit sphere. See [13] for more information.

The product rule for the fractional derivative $\mathbb{D}_{M}^{\alpha} f(x)$ and the fractional gradient $\nabla_{M}^{\alpha} f(x)$ can now be stated and proven using Fourier transforms. The next theorem is the main result of this paper.

TheOrem 3.1. Suppose that $f(x) g(x)$ is integrable and has continuous integrable first order partial derivatives that vanish at infinity if $0<\alpha<1$, or continuous integrable partial derivatives up to order two that vanish at infinity if $1<\alpha<2$. Suppose that, for a fixed $\theta$ on the unit sphere, $\mathbb{D}_{\theta}^{\alpha-j} f(x)$ exists and is of polynomial growth for every integer $j \geq 0, g \in S\left(\mathbb{R}^{d}\right)$, and the series

$$
\sum_{j=0}^{\infty}\left(\begin{array}{l}
\alpha \\
j
\end{array}\right) \mathbb{D}_{\theta}^{\alpha-j} f \mathbb{D}_{\theta}^{j} g
$$


converges in $S^{\prime}\left(\mathbb{R}^{d}\right)$. Then

$$
\mathbb{D}_{\theta}^{\alpha}[f(x) g(x)]=\sum_{j=0}^{\infty}\left(\begin{array}{l}
\alpha \\
j
\end{array}\right) \mathbb{D}_{\theta}^{\alpha-j} f(x) \mathbb{D}_{\theta}^{j} g(x) .
$$

holds in the sense of generalized functions. If the series (3.4) converges pointwise, then (3.5) also holds pointwise. If (3.4) converges uniformly in $\theta$ on the unit sphere, then

$$
\begin{aligned}
& \mathbb{D}_{M}^{\alpha}[f(x) g(x)]=\sum_{j=0}^{\infty}\left(\begin{array}{c}
\alpha \\
j
\end{array}\right) \int_{\|\theta\|=1} \mathbb{D}_{\theta}^{\alpha-j} f(x) \mathbb{D}_{\theta}^{j} g(x) M(d \theta), \\
& \nabla_{M}^{\alpha}[f(x) g(x)]=\sum_{j=0}^{\infty}\left(\begin{array}{c}
\alpha \\
j
\end{array}\right) \int_{\|\theta\|=1} \theta \mathbb{D}_{\theta}^{\alpha-j} f(x) \mathbb{D}_{\theta}^{j} g(x) M(d \theta) .
\end{aligned}
$$

P r o o f. The proof is similar to Theorem 2.1. using Fourier transforms. The Fourier inversion theorem for real-valued functions on $\mathbb{R}^{d}$ is stated in [11, Theorem 1.3.7]: If $\int|h(x)| d x<\infty$, then the Fourier transform $\hat{h}(k)$ exists. Then if $\int|\hat{h}(k)| d k<\infty$, we have

$$
h(x)=(2 \pi)^{-d} \int e^{i k \cdot x} \hat{h}(k) d k
$$

for all $x \in \mathbb{R}^{d}$. Now a substitution $z=k-y$ shows that the inverse Fourier transform of $(2 \pi)^{-d}[\hat{f}(k) * \hat{g}(k)]$ is

$$
\begin{aligned}
& (2 \pi)^{-2 d} \int e^{i k \cdot x}\left(\int \hat{f}(k-y) \hat{g}(y) d y\right) d k \\
& =(2 \pi)^{-2 d} \int e^{i(z+y) \cdot x} \int \hat{f}(z) \hat{g}(y) d y d z \\
& =(2 \pi)^{-d} \int e^{i z \cdot x} \hat{f}(z) d z(2 \pi)^{-d} \int e^{i y \cdot x} \hat{g}(y) d y \\
& =f(x) g(x) .
\end{aligned}
$$

Use the fractional binomial formula $(\underline{2.6})$ to write

$$
(i k \cdot \theta)^{\alpha}=\{(i[k-y] \cdot \theta)+(i y \cdot \theta)\}^{\alpha}=\sum_{j=0}^{\infty}\left(\begin{array}{c}
\alpha \\
j
\end{array}\right)(i[k-y] \cdot \theta)^{\alpha-j}(i y \cdot \theta)^{j}
$$

for any $\alpha>0$. Since $f(x) g(x)$ and its derivatives (of order one if $0<\alpha<1$, or up to order two if $1<\alpha<2$ ) are continuous and integrable and vanish at infinity, the fractional directional derivative $\mathbb{D}_{\theta}^{\alpha}[f(x) g(x)]$ can be defined as the function with Fourier transform $(i k \cdot \theta)^{\alpha}(2 \pi)^{-d}[\hat{f}(k) * \hat{g}(k)]$, see [13, Examples 6.28-6.29]. For a function $\varphi \in S\left(\mathbb{R}^{d}\right)$, write 


$$
\begin{aligned}
& \left\langle\widehat{\mathbb{D}_{\theta}^{\alpha}[f g]}, \varphi\right\rangle \\
& =\int(i k \cdot \theta)^{\alpha}(2 \pi)^{-d}[\hat{f}(k) * \hat{g}(k)] \varphi(k) d k \\
& =\iint \sum_{j=0}^{\infty}\left(\begin{array}{c}
\alpha \\
j
\end{array}\right)(i[k-y] \cdot \theta)^{\alpha-j}(i y \cdot \theta)^{j}(2 \pi)^{-d} \hat{f}(k-y) \hat{g}(y) \varphi(k) d y d k \\
& =\sum_{j=0}^{\infty}\left(\begin{array}{c}
\alpha \\
j
\end{array}\right)(2 \pi)^{-d} \iint(i[k-y] \cdot \theta)^{\alpha-j} \hat{f}(k-y)(i y \cdot \theta)^{j} \hat{g}(y) \varphi(k) d y d k .
\end{aligned}
$$

Continuing as in the proof of Theorem 2.1, using the Fourier inversion formula (3.7), we have

$$
\left\langle\mathbb{D}_{\theta}^{\alpha}[f g], \hat{\varphi}\right\rangle=\sum_{j=0}^{\infty}\left(\begin{array}{c}
\alpha \\
j
\end{array}\right)\left\langle\mathbb{D}_{\theta}^{\alpha-j} f \mathbb{D}_{\theta}^{j} g, \hat{\varphi}\right\rangle .
$$

As in the one-variable case, if the series (3.4) converges in the ordinary sense, then for a fixed $y$ we take $\hat{\varphi}(x)=\omega_{\epsilon}(x-y)$, where

$$
\omega_{\epsilon}(x)=c_{\epsilon} \exp \left\{-\epsilon^{2} /\left(\epsilon^{2}-|x|^{2}\right)\right\} 1_{\{|x|<\epsilon\}},
$$

and constant $c_{\epsilon}$ is chosen so that $\int \omega_{\epsilon}(x) d x=1$. Then for a locally integrable function $h(x), \int \omega_{\epsilon}(x-y) h(y) d y$ converges to $h(x)$ uniformly on compact sets as $\epsilon \rightarrow 0$. Since (3.8) holds for every $\hat{\varphi}(x)=\omega_{\epsilon}(x-y)$, it follows that the product rule (3.5) holds pointwise. Finally, if the series in (3.4) converge uniformly in $\theta$ on the unit sphere, we can apply (3.2) to show that (3.6) holds.

REMARK 3.1. In the special case $\alpha=2$, if the mixing measure $M(d \theta)$ assigns unit mass to the positive coordinate vectors, then the fractional product rule (3.6) reduces to the product rule (3.1) for the Laplacian.

EXAMPLE 3.1. An application to chemical mixing in one dimension was explained in Section 2. In higher dimensions, the local reaction rate

$$
r=\frac{\partial c_{1}}{\partial u} \frac{\partial u}{\partial t}+v \frac{\partial c_{1}}{\partial u} \frac{\partial u}{\partial x}-a \nabla_{M}^{\alpha-1}\left[\frac{\partial c_{1}}{\partial u} \nabla u\right]
$$

and the fractional product rule in Theorem $\mathbf{3 . 1}$ can be applied, along with the semi-analytic solution to the multi-variable FADE (3.3) for $u$ in terms of a multi-variable $\alpha$-stable probability density function [10], to solve for the local reaction rate $r(x, t)$. To apply Theorem 3.1 with $f=\partial c_{1} / \partial u$ and $g=\nabla u$, approximate the stable density $u(x)$ by a tempered stable as in Remark 2.2. 


\section{Acknowledgements}

D.B. was partially supported by NSF Grant EAR-1113704.

M.M.M. was partially supported by NSF grants DMS-1025486, DMS0803360, and NIH grant R01-EB012079.

\section{References}

[1] B. Baeumer and M. M. Meerschaert, Tempered stable Lévy motion and transient super-diffusion. J. Comput. Appl. Math. 233 (2010), 24382448.

[2] D. A. Benson, R. Schumer, M. M. Meerschaert and S. W. Wheatcraft, Fractional dispersion, Lévy motion, and the MADE tracer tests. Transp. Porous Media 42 (2001), 211-240.

[3] D. Bolster, D. A. Benson, T. LeBorgne and M. Dentz, Anomalous mixing and reaction induced by superdiffusive nonlocal transport. Phys. Rev. E 82, 021119.

[4] A. K. Grünwald, Über begrenzte Derivationen und deren Anwedung. Z. Math. Phys. 12 (1867), 441-480.

[5] M. G. Hahn, K. Kobayashi, and S. Umarov, Fokker-Planck-Kolmogorov equations associated with time-changed fractional Brownian motion. Proc. Amer. Math. Soc. 139, No 2 (2011), 691-705.

[6] E. Hille, Analytic Function Theory. Vol. 1. Ginn, Boston (1959).

[7] V. N. Kolokoltsov, Generalized continuous-time random walks, subordination by hitting times, and fractional dynamics. Theory Probab. Appl. 53 (2009), 594-609.

[8] A. V. Letnikov, Theory of differentiation of fractional order. Mat. Sb. 3 (1868), 1-68.

[9] J. Liouville, Memoire sur le calcul des differentielles a indices quelconques. J. de L'Ecole Polytechnique 13 (1832), 71-162.

[10] M. M. Meerschaert, D. A. Benson, and B. Baeumer, Multidimensional advection and fractional dispersion. Phys. Rev. E 59 (1999), 5026-5028.

[11] M. M. Meerschaert and H.-P. Scheffler, Limit Distributions for Sums of Independent Random Vectors: Heavy Tails in Theory and Practice. John Wiley and Sons, New York (2001).

[12] M. M. Meerschaert, J. Mortensen and S. W. Wheatcraft, Fractional vector calculus for fractional advection-dispersion. Physica A $\mathbf{3 6 7}$ (2006), 181-190.

[13] M. M. Meerschaert and A. Sikorskii, Stochastic Models for Fractional Calculus. De Gruyter Studies in Mathematics 43, De Gruyter, Berlin (2012); http://www.degruyter.com/view/product/129781 
[14] T. J. Osler, Leibniz rule for fractional derivatives generalized and an application to infinite series. SIAM. J. Appl. Math. 18 (1970), 658-674.

[15] I. Podlubny, Fractional Differential Equations. Academic Press, New York (1999).

[16] J. Rosiński, Tempering stable processes. Stoch. Proc. Appl. 117 (2007), 677-707.

[17] W. Rudin, Principles of Mathematical Analysis. 3rd edition, McGrawHill (1976).

[18] A. I. Saichev and G. M. Zaslavsky, Fractional kinetic equations: solutions and applications. Chaos 7 (1997), 753-764.

[19] K. Yosida, Functional Analysis. 6th edition, Springer (1980).

${ }^{1}$ Department of Civil Engineering and Geological Sciences

University of Notre Dame

South Bend, IN 46556, USA

e-mail: diogo.bolster.5@nd.edu

2 Department of Statistics and Probability

Michigan State University

East Lansing, MI 48824, USA

e-mail: mcubed@stt.msu.edu

Received: March 29, 2012

3 Department of Statistics and Probability

Michigan State University

East Lansing, MI 48824, USA

e-mail: sikorska@stt.msu.edu

Please cite to this paper as published in:

Fract. Calc. Appl. Anal., Vol. 15, No 3 (2012), pp. 463-478;

DOI: $10.2478 / \mathrm{s} 13540-012-0033-0$ 